\title{
ON WEAK SOLUTION OF A HYPERBOLIC DIFFERENTIAL INCLUSION WITH NONMONOTONE DISCONTINUOUS NONLINEAR TERM
}

\section{GUO XINGMING}

(Received 6 June 1998)

\begin{abstract}
In this paper, a hyperbolic differential inclusion with nonmonotone discontinuous and nonlinear term, which the generalized velocity acts as its variable, is studied and the existence and decay of its weak solution are obtained.

Keywords and phrases. Hyperbolic differential inclusion, nonmonotone discontinuous nonlinear multi-valued system, existence of solution, decay of solution.
\end{abstract}

1991 Mathematics Subject Classification. 34G20.

1. Introduction. In the present paper, we investigate the initial boundary value problem of the following degenerate multi-valued hyperbolic differential inclusion:

$$
\begin{gathered}
\ddot{u}(t)+B(u)(t)+\varphi(\dot{u})(t) \ni f(t), \quad \text { a.e. } t \in[0, T], \\
u(x, t)=0, \quad \text { a.e. }(x, t) \in \sum=\partial \Omega \times[0, T], \\
u(0)=u_{0}, \quad \dot{u}(0)=u_{1},
\end{gathered}
$$

where $B$ is a linear and symmetric operator; $\varphi$ is a discontinuous, nonmonotone, and nonlinear set-valued mapping.

Physical motivations for studying equation (1.1) come partly from problems of continuum mechanics, where nonmonotone, nonlinear, discontinuous, and multi-valued constitutive laws and boundary constraints lead to the above variational inequalities (differential inclusions). For example, when elastobody is constrainted by boundary friction, (1.1) denotes its control equation; if we study viscoelastical body and the unilateral problem of plate, (1.1) is also their control equation, etc. [10, 8, 5].

When $\varphi$ is a nonmonotone multi-valued mapping, generally, for such nonmonotone and discontinuous multi-valued systems, usual monotonicity methods are not valid $[1,6]$. When $\varphi$ degenerates into a class of single-valued mappings and satisfies appropriate conditions, inequation (1.1) become an equation. Equation (1.1) and some of its evolution equations with which it is associated have been investigated and applied intensively [7, 3, 2, 9, 11].

In this paper, we investigate the existence and decay of the weak solutions of the hyperbolic in equation (1.1), with $\varphi$ and $B$ satisfying adequate conditions under zero boundary conditions.

2. Preliminaries. Let $\Omega$ be a bounded open set of $R^{n}$ with regular boundary $\Gamma$. Let $T$ denote a positive real number, $Q=\Omega \times[0, T]$. Suppose that $b \in L_{\mathrm{loc}}^{\infty}(R)$. For every 
$\rho>0$, set

$$
\underline{b}_{p}(\xi)=\underset{\left|\xi_{1}-\xi\right|<p}{\operatorname{ess}} \inf b\left(\xi_{1}\right), \quad \bar{b}_{p}(\xi)=\underset{\left|\xi_{1}-\xi\right|<p}{\operatorname{ess}} \sup b\left(\xi_{1}\right),
$$

and

$$
\underline{b}(\xi)=\lim _{p \rightarrow 0^{+}} \underline{b}_{p}(\xi), \quad \bar{b}(\xi)=\lim _{p \rightarrow 0^{+}} \bar{b}_{p}(\xi), \quad \varphi(\xi)=[\underline{b}(\xi), \bar{b}(\xi)] .
$$

Let $J(\xi)=\int_{0}^{\xi} b(t) d t$. Then $\partial^{c} J(\xi) \subseteq \varphi(\xi)$, where $\partial^{c} J(\xi)$ denotes the Clarke-subdifferential of $J$.

REMARK. If $b\left(\xi_{ \pm}\right)$exists for every $\xi \in R$, then $\varphi(\xi)=\partial^{c} J(\xi)$. Furthermore, if $J$ is convex, $\varphi(\xi)$ is maximal monotone. If $b$ is continuous at $\xi$, then $\varphi(\xi)$ is single-valued at $\xi([3])$.

Let $V=H_{0}^{1}(\Omega),\langle\cdot, \cdot\rangle$ denote the dual pair between $V=H_{0}^{1}(\Omega)$ and $V^{\prime}=H^{-1}(\Omega)$, and $(\cdot, \cdot)$ the inner product of $L^{2}(\Omega)$ which is compatible with the dual pair. Let $|x|_{X}$ denote the norm of an element $x$ of a Banach space $X$.

Consider the following initial boundary value problem of a hyperbolic variational inequation (inclusion):

$$
\begin{gathered}
\ddot{u}(t)+B u(t)+g(t)=f(t), \quad \text { a.e. } t \in[0, T], \\
g(x, t) \in \varphi(\dot{u}(x, t)), \quad \text { a.e. }(x, t) \in Q_{T}=\Omega \times[0, T], \\
u(x, t)=0, \quad \text { a.e. }(x, t) \in \sum=\partial \Omega \times[0, T], \\
u(0)=u_{0}, \quad \dot{u}(0)=u_{1},
\end{gathered}
$$

where $f, u_{0}$, and $u_{1}$ are given.

\section{Existence of solution}

THEOREM 1. Assume that $f \in L^{2}\left(0, T ; L^{2}(\Omega)\right), u_{0} \in H_{0}^{1}(\Omega), u_{1} \in L^{2}(\Omega)$. If

(1) $\exists c>0,|b(\xi)| \leq c(1+|\xi|)$, a.e. $\xi \in R$,

(2) $B: H_{0}^{1}(\Omega) \rightarrow H^{-1}(\Omega)$ is linear, continuous, symmetric, and semicoercive, i.e., $\exists c_{1}>$ $0, c_{2}>0, c_{3} \geq 0$,

$$
\begin{gathered}
|B v|_{H^{-1}(\Omega)} \leq c_{1}|v|_{H_{0}^{1}(\Omega)}, \\
\langle B u, v\rangle=\langle B v, u\rangle \quad \forall u, v \in H_{0}^{1}(\Omega), \\
\langle B v, v\rangle+c_{3}|v|_{L^{2}(\Omega)}^{2} \geq c_{2}|v|_{H_{0}^{1}(\Omega)}^{2} \quad \forall v \in H_{0}^{1}(\Omega),
\end{gathered}
$$

then there exists a function $u$, defined in $\Omega \times[0, T]$, such that

$$
\begin{aligned}
& u \in L^{\infty}\left(0, T ; H_{0}^{1}(\Omega)\right) \cap C\left([0, T] ; L^{2}(\Omega)\right), \\
& \dot{u} \in L^{\infty}\left(0, T ; L^{2}(\Omega)\right) \cap C\left([0, T] ; H^{-1}(\Omega)\right), \\
& \ddot{u} \in L^{2}\left(0, T ; H^{-1}(\Omega)\right),
\end{aligned}
$$

and

$$
\begin{gathered}
\ddot{u}(t)+B u(t)+g(t)=f(t) \quad \text { in } L^{2}\left(0, T ; H^{-1}(\Omega)\right), \\
g(t) \in \varphi(\dot{u}(x, t)), \quad \text { a.e. }(x, t) \in \Omega \times[0, T], \\
u(0)=u_{0}, \quad \dot{u}(0)=u_{1} .
\end{gathered}
$$


Proof. Let $\left\{e_{n}\right\}_{n=1}^{\infty}$ be a subset of $V=H_{0}^{1}(\Omega)$ satisfying $\overline{\operatorname{span}\left\{e_{n}\right\}}=V,\left(e_{i}, e_{j}\right)=\delta_{i j}$. Moreover, let $x_{n}=\sum_{1}^{n} \omega_{i}^{1} e_{i} \rightarrow u_{0}$ strongly in $V, y_{n}=\sum_{1}^{n} \omega_{i}^{2} e_{i} \rightarrow u_{1}$ strongly in $L^{2}(\Omega)$.

Consider the following regularized equation of inequation (1.1)

$$
\ddot{\xi}^{n}=N^{n}+h,\left.\quad \xi^{n}\right|_{t=0}=\omega^{1 n},\left.\quad \dot{\xi}^{n}\right|_{t=0}=\omega^{2 n},
$$

where

$$
\begin{array}{r}
\xi^{n}=\left\{\xi_{i}^{n}\right\}_{1 \times n}, \quad \omega^{1 n}=\left\{\omega_{i}^{1}\right\}_{1 \times n}, \quad \omega^{2 n}=\left\{\omega_{i}^{2}\right\}_{1 \times n}, \quad h=\left\{\left\langle f, e_{i}\right\rangle\right\}_{1 \times n}, \\
N^{n}=\left\{N_{i}^{n}\right\}_{1 \times n}, \quad N_{i}^{n}=-\left\langle B\left(\sum_{1}^{n} \xi_{j}^{n} e_{j}\right), e_{i}\right\rangle-\left\langle b\left(\sum_{1}^{n} \dot{\xi}_{j}^{n} e_{j}\right), e_{i}\right\rangle,
\end{array}
$$

where "." denotes time derivate.

Equation (3.4) is a set of second-order ordinary differential equation and its local solution $\xi^{n}$ exists on $I_{n}=\left[0, T_{n}\right], 0<T_{n} \leq T$.

Set $u_{n}(t)=\sum_{1}^{n} \xi_{j}^{n} e_{j}\left(t \in I_{n}\right)$. Equation (3.4) is equivalent to

$$
\left\langle\ddot{u}_{n}, e_{i}\right\rangle=-\left\langle B u_{n}, e_{i}\right\rangle-\left\langle b\left(\dot{u}_{n}\right), e_{i}\right\rangle+\left\langle f, e_{i}\right\rangle, \quad i=1,2, \ldots, n .
$$

Multiplying equation (3.6) by $\dot{\xi}_{i}^{n}$, summing from $i=1$ to $i=n$, and integrating over $[0, t]\left(t \leq I_{n}\right)$, we get

$$
\begin{aligned}
\left|\dot{u}_{n}(t)\right|_{L^{2}(\Omega)}^{2}+ & \left\langle B u_{n}(t), u_{n}(t)\right\rangle+2 \int_{0}^{t}\left\langle b\left(\dot{u}_{n}\right), \dot{u}_{n}\right\rangle d \tau \\
& =2 \int_{0}^{t}\left\langle f, \dot{u}_{n}\right\rangle d \tau+\left(y_{n}, y_{n}\right)+\left\langle B x_{n}, x_{n}\right\rangle, \\
\left|b\left(\dot{u}_{n}\right)\right|_{L^{2}\left(0, t ; L^{2}(\Omega)\right)}^{2} & =\int_{0}^{t}\left|b\left(\dot{u}_{n}\right)\right|_{L^{2}(\Omega)}^{2} d \tau \leq c \int_{0}^{t} \int_{\Omega}\left(1+\left|\dot{u}_{n}\right|\right)^{2} d x d \tau \\
& \leq \frac{c}{2} \int_{0}^{t}\left(|\Omega|+\left|\dot{u}_{n}(t)\right|_{L^{2}(\Omega)}^{2}\right) d \tau \\
& \leq c_{4}+\frac{c}{2} \int_{0}^{t}\left|\dot{u}_{n}(\tau)\right|_{L^{2}(\Omega)}^{2} d \tau,
\end{aligned}
$$

where $|\Omega|$ denotes the Lebesgue measure of domain $\Omega$.

$$
\begin{aligned}
\int_{0}^{t}\left\langle b\left(\dot{u}_{n}\right), \dot{u}_{n}\right\rangle d \tau & \leq\left|b\left(\dot{u}_{n}\right)\right|_{L^{2}\left(0, t ; L^{2}(\Omega)\right)} \cdot\left|\dot{u}_{n}\right|_{L^{2}\left(0, t ; L^{2}(\Omega)\right)} \\
& \leq \frac{1}{2}\left(\left|b\left(\dot{u}_{n}\right)\right|_{L^{2}\left(0, t ; L^{2}(\Omega)\right)}^{2}+\left|\dot{u}_{n}\right|_{L^{2}\left(0, t ; L^{2}(\Omega)\right)}^{2}\right) \\
& \leq \frac{1}{2}\left\{c_{4}+\left(\frac{c}{2}+1\right) \int_{0}^{t}\left|\dot{u}_{n}(\tau)\right|_{L^{2}(\Omega)}^{2} d \tau\right\}, \\
\int_{0}^{t}\left\langle f, \dot{u}_{n}\right\rangle d \tau & \leq|f|_{L^{2}\left(0, T ; L^{2}(\Omega)\right)} \cdot\left|\dot{u}_{n}\right|_{L^{2}\left(0, t ; L^{2}(\Omega)\right)} \\
& \leq \frac{1}{2}\left(|f|_{L^{2}\left(0, T ; L^{2}(\Omega)\right)}^{2}+\left|\dot{u}_{n}\right|_{L^{2}\left(0, t ; L^{2}(\Omega)\right)}^{2}\right) .
\end{aligned}
$$

From (3.7), $\exists c_{5}>0$ such that

$$
\begin{aligned}
\left|\dot{u}_{n}(t)\right|_{L^{2}(\Omega)}^{2} & +c_{2}\left|u_{n}(t)\right|_{H_{0}^{1}(\Omega)}^{2} \\
& \leq c_{5}+c_{3}\left|u_{n}(t)\right|_{L^{2}(\Omega)}^{2}+\frac{1}{2}\left(\frac{c}{2}+1\right) \int_{0}^{t}\left|u_{n}(\tau)\right|_{L^{2}(\Omega)}^{2} d \tau .
\end{aligned}
$$


We note that

$$
\begin{aligned}
u_{n}(t) & =u_{n}(0)+\int_{0}^{t} \dot{u}_{n} d \tau \\
\left|u_{n}(t)\right|_{L^{2}(\Omega)} & \leq\left|u_{n}(0)\right|_{L^{2}(\Omega)}+\int_{0}^{t}\left|\dot{u}_{n}\right|_{L^{2}(\Omega)} d \tau .
\end{aligned}
$$

By Hölder inequality, $\exists c_{6}, c_{7}>0$ such that

$$
\left|u_{n}(t)\right|_{L^{2}(\Omega)}^{2} \leq c_{6}+c_{7} \int_{0}^{t}\left|\dot{u}_{n}(\tau)\right|_{L^{2}(\Omega)}^{2} d \tau .
$$

From (3.10) and (3.12), we obtain: $\exists c_{8}, c_{9}>0$ such that

$$
\left|\dot{u}_{n}(t)\right|_{L^{2}(\Omega)}^{2}+c_{2}\left|u_{n}(t)\right|_{H_{0}^{1}(\Omega)}^{2} \leq c_{8}+c_{9} \int_{0}^{t}\left|\dot{u}_{n}(\tau)\right|_{L^{2}(\Omega)}^{2} d \tau, \quad\left(t \in I_{n}\right) .
$$

Hence,

$$
\left|\dot{u}_{n}(t)\right|_{L^{2}(\Omega)}^{2} \leq c_{8}+c_{9} \int_{0}^{t}\left|\dot{u}_{n}(\tau)\right|_{L^{2}(\Omega)}^{2} d \tau, \quad\left(t \in I_{n}\right)
$$

By Gronwall's inequality, we have

$$
\left|\dot{u}_{n}(t)\right|_{L^{2}(\Omega)}^{2} \leq c_{8} \exp \left(c_{9} t\right), \quad\left(t \in I_{n}\right) .
$$

Therefore, from (3.12), (3.15), and (3.16), there exists $c_{10}>0$ such that

$$
\left|\dot{u}_{n}(t)\right|_{L^{2}(\Omega)} \leq C_{10}, \quad\left|u_{n}(t)\right|_{L^{2}(\Omega)} \leq C_{10}, \quad\left|u_{n}(t)\right|_{H_{0}^{1}(\Omega)} \leq C_{10}, \quad\left(t \in I_{n}\right),
$$

where $c_{4}, c_{5}, c_{6}, c_{7}, c_{8}, c_{9}, c_{10}$ are positive constants independent of $n$ and $T_{n}$. The estimate (3.16) implies that we can prolongate the solution of equation (3.4) to the interval $[0, T]$, i.e., $I_{n}=[0, T](\forall n)$.

From (3.6), we see that, for every $\eta \in \operatorname{span}\left\{e_{1}, e_{2}, \ldots, e_{n}\right\}$,

$$
\begin{aligned}
\left|\left\langle\ddot{u}_{n}, \eta\right\rangle\right| \leq & |f(t)|_{L^{2}(\Omega)} \cdot|\eta|_{L^{2}(\Omega)}+\left|b\left(\dot{u}_{n}\right)\right|_{L^{2}(\Omega)} \cdot|\eta|_{L^{2}(\Omega)} \\
& +|B| \cdot\left|u_{n}\right|_{H_{0}^{1}(\Omega)} \cdot|\eta|_{H_{0}^{1}(\Omega)},
\end{aligned}
$$

where $|B|$ is the norm of the linear continuous operator $B$.

$$
\begin{aligned}
\left|\ddot{u}_{n}(t)\right|_{H^{-1}(\Omega)} & =\sup _{|\eta|_{V}=1}\left|\left\langle\ddot{u}_{n}(t), \eta\right\rangle\right|=\sup _{\substack{\left.\eta \in \operatorname{span}_{\left.\mid \eta e_{1}, \ldots, e_{n}\right\}}|| \eta\right|_{V}=1}}\left|\left\langle\ddot{u}_{n}(t), \eta\right\rangle\right| \\
& \leq c_{11}\left(|f(t)|_{L^{2}(\Omega)}+\left|b\left(\dot{u}_{n}\right)\right|_{L^{2}(\Omega)}\right)+|B| \cdot\left|u_{n}(t)\right|_{H_{0}^{1}(\Omega)},
\end{aligned}
$$

where $c_{11}$ is the imbedding constant which $H_{0}^{1}(\Omega)$ imbeds in $L^{2}(\Omega)$.

$$
\left|b\left(\dot{u}_{n}\right)(t)\right|_{L^{2}(\Omega)}^{2} \leq c \int_{\Omega}\left(1+\left|\dot{u}_{n}(t)\right|\right)^{2} d x \leq \frac{c}{2}\left(|\Omega|+\left|\dot{u}_{n}(t)\right|_{L^{2}(\Omega)}^{2}\right) .
$$

This shows that $\left\{b\left(\dot{u}_{n}\right)\right\}$ is also a bounded subset of $L^{\infty}\left(0, T ; L^{2}(\Omega)\right)$. Hence, (3.18) implies that $\left\{\ddot{u}_{n}\right\}$ is a bounded subset of $L^{2}\left(0, T ; H^{-1}(\Omega)\right)$. 
Therefore, there exists a subsequence of $\left\{u_{n}\right\}$ (still denoted by itself) and a function $u$ such that $u \in L^{\infty}\left(0, T ; H_{0}^{1}(\Omega)\right), \dot{u} \in L^{\infty}\left(0, T ; L^{2}(\Omega)\right), \ddot{u} \in L^{2}\left(0, T ; H^{-1}(\Omega)\right)$ satisfying

$$
\begin{aligned}
& u_{n} \rightarrow u \text { weakly-star in } L^{\infty}\left(0, T ; H_{0}^{1}(\Omega)\right), \\
& \dot{u}_{n} \longrightarrow \dot{u} \text { weakly-star in } L^{\infty}\left(0, T ; L^{2}(\Omega)\right), \\
& \ddot{u}_{n} \longrightarrow \ddot{u} \quad \text { weakly in } L^{2}\left(0, T ; H^{-1}(\Omega)\right), \\
& b\left(\dot{u}_{n}\right) \longrightarrow g \quad \text { weakly-star in } L^{\infty}\left(0, T ; L^{2}(\Omega)\right) .
\end{aligned}
$$

Furthermore, $\dot{u}_{n}(t, x) \rightarrow \dot{u}(t, x)$, a.e. $(t, x) \in[0, T] \times \Omega$.

It is well known that the space $W(V)$, defined by $W(V)=\left\{u \in L^{2}(0, T ; V), \dot{u} \in\right.$ $\left.L^{2}\left(0, T ; V^{\prime}\right)\right\}$ with the norm $|u|_{W}=|u|_{L^{2}(0, T ; V)}+|\dot{u}|_{L^{2}\left(0, T ; V^{\prime}\right)}$, is continuously imbedded in $C\left([0, T] ; L^{2}(\Omega)\right)$. It is obvious that $u \in C\left(0, T ; L^{2}(\Omega)\right), \dot{u} \in C\left(0, T ; H^{-1}(\Omega)\right)$. Hence, $u(0), \dot{u}(0)$ make sense.

For $\lambda \in L^{2}(0, T)$, by (3.6), we have

$$
\begin{aligned}
\int_{0}^{T}\left\langle\ddot{u}_{n}, \lambda e_{i}\right\rangle d t= & -\int_{0}^{T}\left\langle B\left(u_{n}\right), \lambda e_{i}\right\rangle d t-\int_{0}^{T}\left\langle b\left(\dot{u}_{n}\right), \lambda e_{i}\right\rangle d t \\
& +\int_{0}^{T}\left\langle f(t), \lambda e_{i}\right\rangle d t, \quad i=1,2, \ldots, n .
\end{aligned}
$$

For every given positive integer $i$, let $n \rightarrow \infty$ in (3.21). Then, it follows that

$$
\begin{aligned}
\int_{0}^{T}\left\langle\ddot{u}, \lambda e_{i}\right\rangle d t= & -\int_{0}^{T}\left\langle B(u), \lambda e_{i}\right\rangle d t-\int_{0}^{T}\left\langle g, \lambda e_{i}\right\rangle d t \\
& +\int_{0}^{T}\left\langle f(t), \lambda e_{i}\right\rangle d t, \quad i=1,2, \ldots
\end{aligned}
$$

Therefore,

$$
\ddot{u}(t)+B(u)+g(t)=f(t) \quad \text { in } L^{2}\left(0, T ; H^{-1}(\Omega)\right) .
$$

In the following, we show that

$$
g(x, t) \in \varphi(\dot{u}(x, t)), \quad \text { a.e. }(x, t) \in Q_{T}=\Omega \times[0, T] .
$$

Since $\dot{u}_{n}(x, t) \rightarrow \dot{u}(x, t)$, a.e. $(x, t) \in Q_{T}$, by Eropob's theorem [12], for every $\delta>0$, there exists a subset $Q_{\delta} \subseteq Q_{T}=\Omega \times[0, T]$, $\left|Q_{\delta}\right| \leq \delta$,

$$
\dot{u}_{n}(x, t) \rightarrow \dot{u}(x, t) \text { uniformly in } Q_{T} / Q_{\delta} .
$$

That is, for every $\varepsilon>0$, there exists a positive integer $\bar{N}$, when $n \geq \bar{N}$,

$$
\left|\dot{u}_{n}(x, t)-\dot{u}(x, t)\right| \leq \varepsilon \quad \forall(x, t) \in Q_{T} / Q_{\delta} .
$$

It is obvious that

$$
\underline{b}_{\varepsilon}(\dot{u}(x, t)) \leq b\left(\dot{u}_{n}(x, t)\right) \leq \bar{b}_{\varepsilon}(\dot{u}(x, t)) \quad \forall(x, t) \in Q_{T} / Q_{\delta} .
$$

For every $\mu \in L^{1}\left(0, T ; L^{2}(\Omega)\right), \mu \geq 0$

$$
\begin{aligned}
\int_{Q_{T} \backslash Q_{\delta}} g(x, t) \mu(x, t) d x d t & =\lim _{n \rightarrow \infty} \int_{Q_{T} \backslash Q_{\delta}} b\left(\dot{u}_{n}(x, t)\right) \mu(x, t) d x d t \\
& \leq \int_{Q_{T} \backslash Q_{\delta}} \bar{b}_{\varepsilon}(\dot{u}(x, t)) \mu(x, t) d x d t,
\end{aligned}
$$




$$
\begin{aligned}
\int_{Q_{T} \backslash Q_{\delta}} g(x, t) \mu(x, t) d x d t & \leq \limsup _{\varepsilon \rightarrow 0^{+}} \int_{Q_{T} \backslash Q_{\delta}} \bar{b}_{\varepsilon}(\dot{u}(x, t)) \mu(x, t) d x d t \\
& \leq \int_{Q_{T} \backslash Q_{\delta}} \bar{b}(\dot{u}(x, t)) \mu(x, t) d x d t .
\end{aligned}
$$

Analogously, we can obtain

$$
\int_{Q_{T} \backslash Q_{\delta}} g(x, t) \mu(x, t) d x d t \geq \int_{Q_{T} \backslash Q_{\delta}} \underline{b}(\dot{u}(x, t)) \mu(x, t) d x d t .
$$

Hence,

$$
g(x, t) \in \varphi(\dot{u}(x, t)), \quad \text { a.e. }(x, t) \in Q_{T} / Q_{\delta} .
$$

Letting $\delta \longrightarrow 0^{+}$, we get

$$
g(x, t) \in \varphi(\dot{u}(x, t)), \quad \text { a.e. }(x, t) \in Q_{T}=\Omega \times[0, T] .
$$

Let $\lambda \in C^{1}[0, T], \lambda(T)=0$. Integrating by parts the left-hand sides of equations (3.21) and (3.22) gives

$$
\begin{array}{r}
-\left\langle\dot{u}_{n}(0), \lambda(0) e_{i}\right\rangle-\int_{0}^{T}\left\langle\dot{u}_{n}, \dot{\lambda} e_{i}\right\rangle d t=\text { the right of (3.21), } \\
-\left\langle\dot{u}(0), \lambda(0) e_{i}\right\rangle-\int_{0}^{T}\left\langle\dot{u}, \dot{\lambda} e_{i}\right\rangle d t=\text { the right of (3.22). }
\end{array}
$$

Making a comparison between the two equations of (3.33), we get

$$
\lim _{n \rightarrow \infty}\left\langle\dot{u}_{n}(0)-\dot{u}(0), e_{i}\right\rangle=0, \quad i=1,2, \ldots
$$

Therefore,

$$
\dot{u}_{n}(0) \longrightarrow \dot{u}(0) \text { weakly in } H^{-1}(\Omega) .
$$

The uniqueness of the limit implies that $\dot{u}(0)=u_{1}$ (in $H^{-1}(\Omega)$ ).

Let $\lambda \in C^{2}[0, T], \lambda(T)=0, \dot{\lambda}(T)=0$. Analogously, integrating by parts the left-hand sides of equations (3.33), and making a comparison with the obtained results again gives: $u(0)=u_{0}\left(\right.$ in $\left.L^{2}(\Omega)\right)$. This completes the proof.

THEOREM 2. Let $f \in L^{2}\left(0, T ; L^{2}(\Omega)\right), u_{0} \in H_{0}^{1}(\Omega), u_{1} \in L^{2}(\Omega)$. Assume that $b$ satisfies $(1)^{\prime} b(\xi) \xi \geq-\delta$ for almost everywhere $\xi \in R$, and $\exists \bar{c}>0,|b(\xi)| \leq \bar{c}\left(1+|\xi|^{p}\right)$, a.e. $\xi \in R$, if $n>2,0<p \leq(2 n) /(n-2)$; if $n \leq 2,0 \leq p<\infty$, and condition (2) of Theorem 1 is valid. Then there exists a function $v$, defined in $\Omega \times[0, T]$, satisfying

$$
v \in L^{\infty}\left(0, T ; H_{0}^{1}(\Omega)\right), \quad \dot{v} \in L^{\infty}\left(0, T ; L^{2}(\Omega)\right),
$$

and

$$
\begin{gathered}
\ddot{v}+B(v)+\bar{g}(t)=f(t) \quad \text { in } L^{1}\left(0, T ; H^{-1}(\Omega)+L^{1}(\Omega)\right), \\
\bar{g}(x, t) \in \varphi(\dot{v}(x, t)), \quad \text { a.e. }(x, t) \in Q_{T}=\Omega \times[0, T], \\
v(0)=u_{0}, \quad \dot{v}(0)=u_{1} .
\end{gathered}
$$


Proof. Analogously to Theorem 1, we still may get (3.7), where $\left\{e_{n}\right\}_{n=1}^{\infty}$ is a basis of $H_{0}^{1}(\Omega) \cap L^{\infty}(\Omega)$ satisfying $\left(e_{i}, e_{j}\right)=\delta_{i j}$. Under assumption $(1)^{\prime}, \int_{0}^{t}\left\langle b\left(\dot{u}_{n}\right), \dot{u}_{n}\right\rangle d \tau \geq-\delta$. From (3.7), we have

$$
\left|\dot{u}_{n}(t)\right|_{L^{2}(\Omega)}^{2}+c_{2}\left|u_{n}(t)\right|_{H_{0}^{1}(\Omega)}^{2} \leq c_{4}+c_{3}\left|u_{n}(t)\right|_{L^{2}(\Omega)}^{2}+2 \int_{0}^{t}\left\langle f, \dot{u}_{n}\right\rangle d \tau .
$$

It is easy to see that equations (3.12), (3.13), (3.15), and (3.16) are still true and the solution of equation (3.4) may still be extended to the interval $[0, T]$.

By Sobolev Imbedding Theorem, we have, for a.e. $t \in[0, T]$, if $n>2$, then

$$
H_{0}^{1}(\Omega) \subset L^{p^{*}}(\Omega) \subset L^{p}(\Omega), \quad p^{*}=\frac{2 n}{n-2},
$$

and if $n=2$, then

$$
H_{0}^{1}(\Omega) \subset L^{q}(\Omega) \quad \forall 1 \leq q \leq \infty,
$$

so

$$
\left|u_{n}(t)\right|_{L^{p}(\Omega)} \leq c_{11}\left|u_{n}(t)\right|_{H_{0}^{1}(\Omega)} \leq c_{11} c_{9}
$$

if $n=1$, then

$$
H_{0}^{1}(\Omega) \subset C(\bar{\Omega}) \text {, and ditto, }\left|u_{n}(t)\right|_{C(\bar{\Omega})}=\max _{x \in \bar{\Omega}}\left|u_{n}(x, t)\right| \leq c_{11} c_{9},
$$

where $\bar{\Omega}$ denotes the Closure of $\Omega$ and $c_{11}$ is the imbedding constant which $H_{0}^{1}(\Omega)$ imbeds in $L^{p}(\Omega)$ or $C(\bar{\Omega})$. Everyway, we always have that $b\left(\dot{u}_{n}\right) \in L^{\infty}\left(0, T ; L^{1}(\Omega)\right)$ and $\left\{b\left(\dot{u}_{n}\right)\right\}$ is a bounded subset of $L^{\infty}\left(0, T ; L^{1}(\Omega)\right)$.

Therefore, there exists a subsequence of $\left\{u_{n}\right\}$, still denoted by itself, and a function $v$, such that $v \in L^{\infty}\left(0, T ; H_{0}^{1}(\Omega)\right), \dot{v} \in L^{\infty}\left(0, T ; L^{2}(\Omega)\right)$, satisfying

$$
\begin{aligned}
u_{n} \longrightarrow v & \text { weakly-star in } L^{\infty}\left(0, T ; H_{0}^{1}(\Omega)\right), \\
\dot{u}_{n} \longrightarrow \dot{v} & \text { weakly-star in } L^{\infty}\left(0, T ; L^{2}(\Omega)\right), \\
b\left(\dot{u}_{n}\right) \longrightarrow \bar{g} & \text { weakly-star in } L^{\infty}\left(0, T ; L^{1}(\Omega)\right) .
\end{aligned}
$$

Since the dual of the space $H_{0}^{1}(\Omega) \cap L^{\infty}(\Omega)$ is the space $H^{-1}(\Omega)+L^{1}(\Omega)$, by (3.6), it is easy to obtain

$$
\ddot{v}(t)+B(v)+\bar{g}(t)=f(t) \quad \text { in } L^{1}\left(0, T ; H^{-1}(\Omega)+L^{1}(\Omega)\right) .
$$

The rest is analogous to that of Theorem 1 .

This completes the proof.

\section{Decay of solution}

TheOREM 3. Let $T=+\infty, f \equiv 0$. Suppose that $\langle B w, w\rangle \geq 0, \forall w \in H_{0}^{1}(\Omega)$. If $(b(w)$, $w) \geq \mu_{0}|w|_{L^{2}(\Omega)}^{2}$, then, under the conditions of Theorem 2, the solution in Theorem 2, obtained from the regularized equation (3.4), satisfies

$$
|\dot{u}(t)|_{L^{2}(\Omega)}^{2} \leq \mu_{1} \exp \left(-\mu_{2} t\right), \text { a.e. } t \geq 0,
$$

where $\mu_{0}, \mu_{1}$, and $\mu_{2}$ are positive constants. 
Proof. Let $u_{n}$ be a solution of (3.4), i.e., $u_{n}$ satisfies (3.6) and (3.7). Since ( $\left.b(w), w\right)$ $\geq \mu_{0}|w|_{L^{2}(\Omega)}^{2}$, by (3.7), we have

$$
\left|\dot{u}_{n}(t)\right|_{L^{2}(\Omega)}^{2}+\left\langle B u_{n}(t), u_{n}(t)\right\rangle \leq c_{12}-2 \mu_{0} \int_{0}^{t}\left|\dot{u}_{n}(\tau)\right|_{L^{2}(\Omega)}^{2} d \tau, \quad t \in[0,+\infty),
$$

where $c_{12}$ is a positive constant independent of $n$.

If $\langle B w, w\rangle \geq 0, \forall w \in H_{0}^{1}(\Omega)$ and $\left\langle B u_{n}(t), u_{n}(t)\right\rangle \geq 0$, then, by Gronwall inequality,

$$
\left|\dot{u}_{n}(t)\right|_{L^{2}(\Omega)}^{2} \leq c_{12} \exp \left(-2 \mu_{0} t\right), \quad \text { a.e. } t \geq 0 .
$$

Since

$$
\left|\dot{u}_{n}(t)\right|_{L^{2}(\Omega)} \leq c_{9}, \quad \dot{u}_{n} \longrightarrow \dot{u} \text { weakly-star in } L^{\infty}\left(0, \infty ; L^{2}(\Omega)\right),
$$

it is easy to obtain that $\dot{u}_{n}(t) \rightarrow \dot{u}(t)$ weakly in $L^{2}(\Omega)$ for almost everywhere $t \geq 0$. But $L^{2}(\Omega)$ is a real Hilbert space, hence, $|\dot{u}(t)|_{L^{2}(\Omega)} \leq \underline{\lim }_{n \rightarrow \infty}\left|\dot{u}_{n}(t)\right|_{L^{2}(\Omega)}$, a.e. $t \geq 0$ (see [4]). Finally, we get

$$
|\dot{u}(t)|_{L^{2}(\Omega)}^{2} \leq c_{12} \exp \left(-2 \delta_{0} t\right), \quad(\text { a.e. } t \geq 0) .
$$

REMARK 1. If $B u=-\Delta u, \varphi(u)=|u|^{p} u$ then (1.1) is the equation which was ever considered by J. L. Lions [6]. J. L. Lions ever obtained the existence and uniqueness. But at this case, the result of decay of solution is true since the conditions of Theorem 3 is satisfied.

REMARK 2. When $B u=-\Delta u$ and $\varphi$ denotes the friction potential, equation (1.1) was considered by P. D. Panagiotopoulos under stronger conditions [8].

ACKNOWLEDGEMENT. This work is supported in part by Natural Science Foundation of China.

\section{REFERENCES}

[1] H. Brezis, Monotonicity methods in Hilbert spaces and some applications to nonlinear partial differential equations, Contributions to nonlinear functional analysis (New York) (E. H. Zarautonelle, ed.), Academic Press, 1971, pp. 101-156. MR 5215126. Zbl 278.47033.

[2] S. Carl and S. Heikkila, An existence result for elliptic differential inclusions with discontinuous nonlinearity, Nonlinear Anal. TMA 18 (1992), no. 5, 471-479. MR 92m:35268. Zbl 755.35039.

[3] K. C. Chang, Variational methods for nondifferentiable functionals and their applications to partial differential equations, J. Math. Anal. Appl. 80 (1981), no. 1, 102-129. MR 82h:35025. Zbl 487.49027.

[4] K. C. Chang and L. Yuanqu, A Course in Functional Analysis (I), Pecking University Press, 1987.

[5] G. Duvaut and J. L. Lions, Les inequations en mecanique et en physique, Travaux et Recherches Mathematiques, vol. 21, Dunod, Paris, 1972. MR 574778. Zbl 298.73001.

[6] A. Haraux, Nonlinear Evolution Equations-Global Behavior of Solutions, Lecture Notes in Mathematics, vol. 841, Springer-Verlag, Berlin, New York, 1981. MR 83d:47066. Zbl 461.35002 . 
[7] J. L. Lions, Quelques methodes de resolution des problemes aux limites nonlineaires, Dunod; Gauthier-Villars, Paris, 1969. MR 41\#4326. Zbl 189.40603.

[8] P. D. Panagiotopoulos, Inequality Problems in Mechanics and Applications, Birkhauser Boston, Inc., Boston, 1985, Convex and nonconvex energy functions. MR 88h:49003. Zbl 579.73014.

[9] _ Coercive and semicoercive hemivariational inequalities, Nonlinear Anal. TMA 16 (1991), no. 3, 209-231. MR 92m:47138. Zbl 733.49012.

[10] _ Hemivariational Inequalities, Springer-Verlag, Berlin, 1993, Applications in mechanics and engineering. MR 97c:73001. Zbl 826.73002.

[11] J. Rauch, Discontinuous semilinear differential equations and multiple valued maps, Proc. Amer. Math. Soc. 64 (1977), no. 2, 277-282. MR 56 835. Zbl 413.35031.

[12] M. Q. Zhou, Real Functions, Pecking University Press, 1985.

XiNGMING: SHANGHAi UNIVERSiTY, SHANGHAi INSTITUTE OF APPLIED MATHEMATICS AND MECHANICS, SHANGHAI 200072, CHINA 


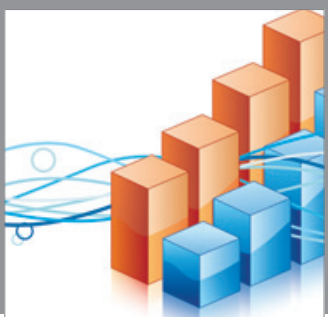

Advances in

Operations Research

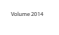

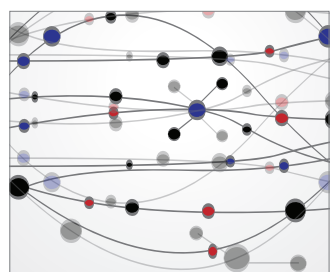

\section{The Scientific} World Journal
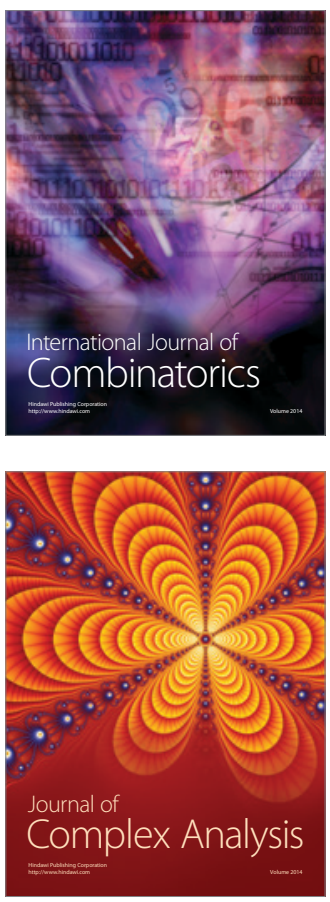

International Journal of

Mathematics and

Mathematical

Sciences
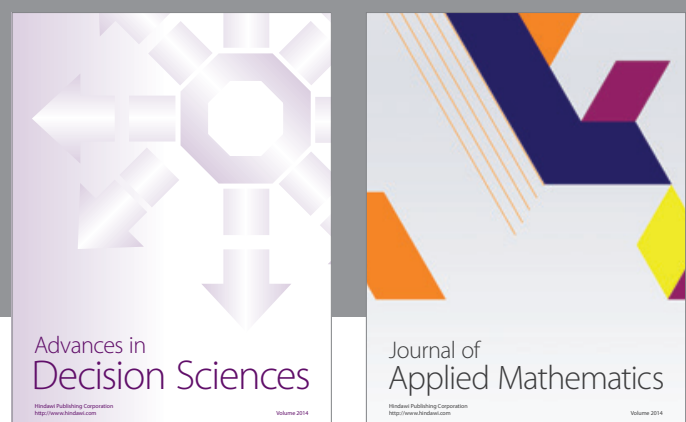

Journal of

Applied Mathematics
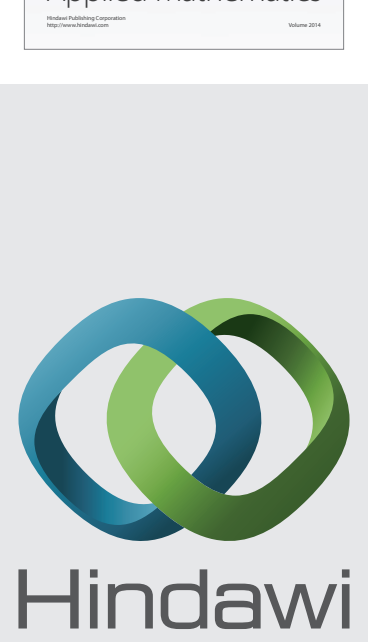

Submit your manuscripts at http://www.hindawi.com
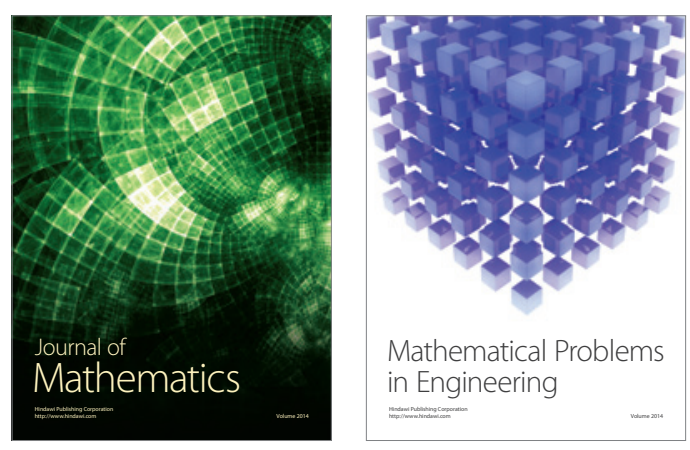

Mathematical Problems in Engineering
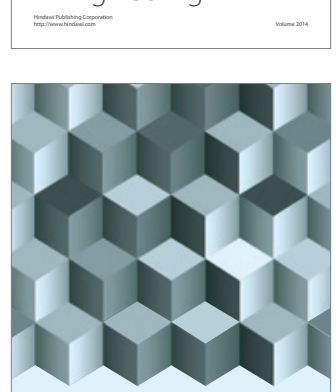

Journal of

Function Spaces
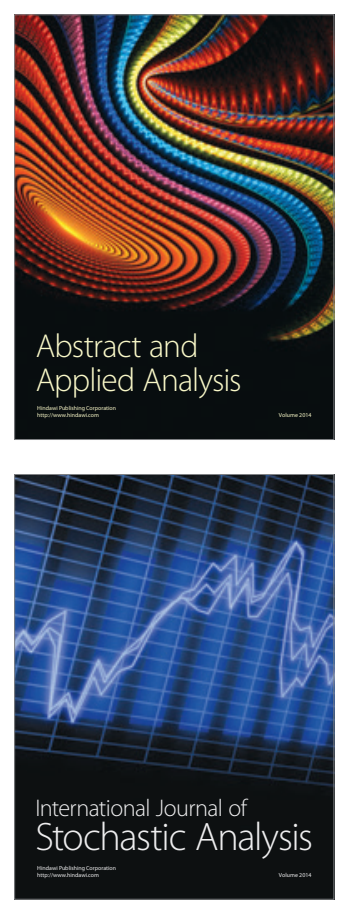

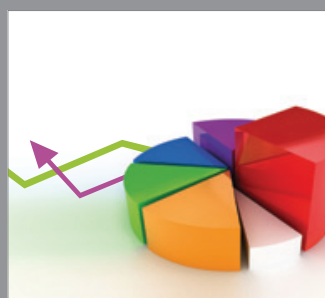

ournal of

Probability and Statistics

Promensencen
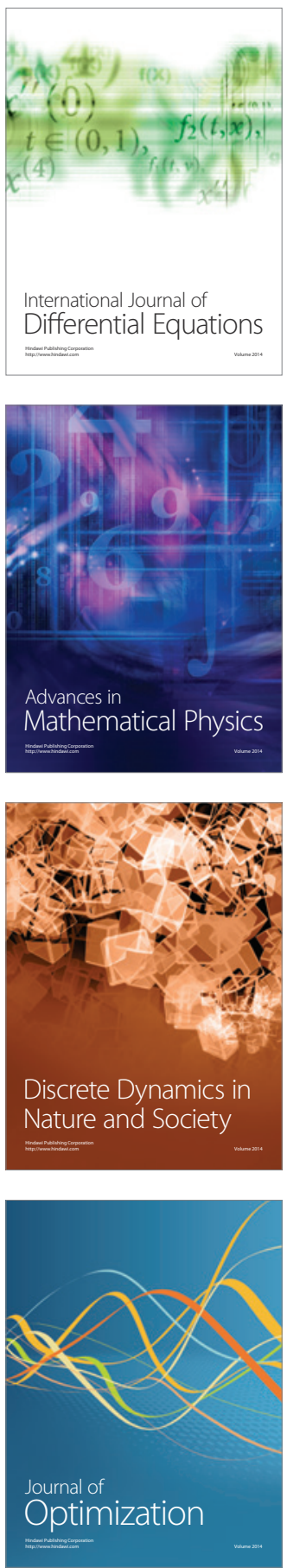